\title{
Stable laser-Doppler flow-motion patterns in the human cutaneous microcirculation: Implications for prospective geroscience studies
}

\author{
V. TÓTH-SZÜKI ${ }^{1}$, F. BARI ${ }^{2}$ and F. DOMOKI ${ }^{1 *}$ (D) \\ ${ }^{1}$ Department of Physiology, Faculty of Medicine, University of Szeged, Szeged, Hungary \\ 2 Department of Medical Physics and Informatics, Faculty of Medicine, University of Szeged, Szeged, \\ Hungary
}

Received: December 9, 2019 • Accepted: February 25, 2020

Published online: April 7, 2020

(c) 2020 The Author(s)

\begin{abstract}
Microvascular function has been assessed by determining the rhythmic oscillations in blood flow induced by the vasomotion of resistance vessels. Although laser-Doppler flowmetry (LDF) allows simple, noninvasive evaluation of this flow-motion in the cutaneous microcirculation, the temporal and spatial reproducibility of such assessments remains unclear.

In the present study, we investigated cutaneous flow-motion in three consecutive years in eight skin regions using LDF in six healthy young volunteers. The characteristic flow-motion frequency was determined using fast-Fourier transformation. Additionally, in two years a more traditional measure of microvascular reactivity, postocclusive reactive hyperemia (PORH) was evoked in the forearm after transient brachial artery occlusion (1-2-3 min) induced by cuff inflation.

Well-defined flow-motion was found in six regions showing significant differences in frequency: the highest flow-motion frequency was found in the frontal and temporal regions $(8.0 \pm 1.1$ and $8.5 \pm 1.0$ cycles/min, cpm, respectively, mean $\pm \mathrm{SD}$ ) followed by the scapular, infraclavicular and coxal regions (7.5 $\pm 1.3 ; 6.7 \pm 1.1$ and $6.5 \pm 1.2 \mathrm{cpm}$, respectively). The lowest, stable flow-motion was found in the posterior femoral region $(5.5 \pm 1.0 \mathrm{cpm})$, whereas flow-motion was detectable only sporadically in the limbs. The region-dependent flow-motion frequencies were very stable within individuals either between the body sides, or among the three measurements, only the infraclavicular region showed a small difference (114 \pm $17 \% *, \%$ of value in 1 st year; $\left.{ }^{*} P<0.05\right)$. However, PORH indices differed after $2-3$ min occlusions significantly in consecutive years.
\end{abstract}

\footnotetext{
* Corresponding author. Department of Physiology, Faculty of Medicine, University of Szeged, 10 Dóm tér, Szeged, 6720, Hungary. Tel.: +3662 545923; Fax: +3662 545842, E-mail: domoki.ferenc@med.u-szeged.hu
} 
We report that flow-motion frequencies determined from LDF signals show both region-specificity and excellent intra-individual temporal and spatial reproducibility suggesting their usefulness for non-invasive follow-up of microvascular reactivity.

\section{KEYWORDS}

vasomotion, postocclusive hyperemia, laser-Doppler flowmetry, cutaneous blood flow, microvascular reactivity

\section{INTRODUCTION}

Microvascular dysfunction is a common denominator in a number of chronic systemic diseases playing a pivotal role in the development of organ damage $[9,14,19]$. The study of the cutaneous circulation with laser-Doppler flowmetry (LDF) in humans offers a straightforward, plausible, noninvasive method to gain insight into the functional status of the microcirculatory bed $[11,18]$. However, the regulation of cutaneous blood flow $(\mathrm{CuBF})$ is uniquely dependent on thermoregulatory needs, therefore the relative importance of local vascular regulatory mechanisms determined for instance by pharmacological approaches cannot likely apply directly to other organ circulations. In contrast to relative flow changes in response to various stimuli that bear organ-specific features, the spectral analysis of the LDF signal reveals rhythmic oscillations in CuBF of different frequencies representing flow-motion [6, 20]. This flow-motion stems from the vasomotion of resistance vessels, and the presence and degree of this vasomotion in the respective frequency ranges have been suggested to provide information on autonomic vasomotor tone, vascular smooth muscle function, and perhaps most importantly on the functional integrity of the microvascular endothelium [20]. The prospective study of microvascular function would be important for tackling aging-related alterations, such as region-specific changes in sympathetic nervous system activity [2], or the functional consequence of mitochondrial impairments in aging microvessels [21]. Also, the efficacy of therapeutic interventions combatting microvascular degeneration could be verified [12]. However, there are numerous unresolved issues concerning the applicability of the study of CuBF flow-motion as a potential index of microvascular health. For instance, it is unclear how many times such a test should be performed to sufficiently characterize the functional status of the microvessels. It is also of utmost importance whether the anatomical location of the assessed skin region has a measurable impact on the determination of flow-motion. To address these issues, the characteristic frequency of CuBF flow-motion was determined in healthy human subjects over the course of three years in multiple skin regions, and thus side-dependence and/or regional differences in flow-motion were also investigated. To be able to compare the stability of our flow-motion frequency data with that of a traditional microvascular response, postocclusive reactive hyperemia (PORH) of the forearm CuBF was also determined.

\section{MATERIALS AND METHODS}

\section{Subjects}

Healthy young volunteers (two males and four females, $20 \pm 1$ years old in the first year of the study) were enrolled in the study after they had been informed about the aim of the study and 
the experimental protocol, and they had given their verbal consent. The subjects were all nonsmokers, did not take any drugs on a regular basis or at the time of the measurements, and had no history of any previous cardiovascular disease or diabetes mellitus. The experimental protocol described below was performed three times, in three consecutive years in all participants, except that PORH was not determined in the third year. The study protocol was approved by the Institutional Human Biomedical Research Ethics Committee (No.: 1454).

\section{Experimental protocol}

CuBF was assessed with two-channel LDF (Periflux 4001, Perimed, Stockholm, Sweden). The LDF probes (PF 408) used in the study possess $250 \mu \mathrm{m}$ fiber separation distances designed for $\mathrm{CuBF}$ measurements. The subjects were lying in a comfortable supine position in a temperaturecontrolled room $\left(23 \pm 0.5{ }^{\circ} \mathrm{C}\right)$, wearing light clothing. After $15 \mathrm{~min}$ acclimatization to reduce anxiety, pulse rate was determined $(69 \pm 91 / \mathrm{min})$, and the arterial blood pressure was measured by sphygmomanometry (RR: $113 \pm 9 / 70 \pm 9 \mathrm{mmHg}$ ). The LDF probes were placed in a sequential order over the following regions: forehead (one probe placed in the midline), temporal region, scapular region, infraclavicular region, coxal region, posterior femoral region, crural region, and the forearm region (2-2 flow probes placed symmetrically on the two sides). Care was taken to avoid visible subcutaneous veins when placing the LDF probes. At each site, the $\mathrm{CuBF}$ was recorded for $10 \mathrm{~min}$ (Fig. $1 \mathrm{~A}$ and B) with a sampling frequency of $32 \mathrm{~Hz}$ and a time constant of 0.2. (PeriSoft 2.0, Perimed, Stockholm, Sweden). Afterward, graded PORH was evoked on the right forearm by inflating the cuff of the sphygmomanometer $30 \mathrm{mmHg}$ above the previously determined systolic blood pressure for 1, 2, and $3 \mathrm{~min}$ followed by 10-10 $\mathrm{min}$ reperfusion elicited with rapid deflation of the cuff. The recorded LDF data were analyzed offline.

\section{LDF data analysis}

The LDF data were analyzed with the Perisoft 2.0 software package. The flow values were subjected to spectral analysis by using fast Fourier transformation (FFT; Fig. 1C and D). For spectral analysis, all data after 10 minutes collected from all the measured regions were used (frontal region, temporal region, scapular region, infraclavicular region, coxal region, posterior femoral region, crural region, and the forearm region). Frequency domain detection was made of the power spectra. In the frequency range specific for vasomotion, between 4 and $12 \mathrm{cpm}$ $(0.67-0.20 \mathrm{~Hz})$ all the peaks were analyzed. After checking for body side- and study yeardependent differences, all data were pooled to search for regional differences. The PORH index is defined as the ratio of the area under the CuBF curve (AUC) $1 \mathrm{~min}$ after the release of the cuff relative to the AUC of a 1-min period before cuff inflation. The PORH indices were calculated for the 1,2, and 3 min occlusions. In addition, the peak CuBF values were determined and expressed as the percent of baseline.

\section{Statistics}

Data are presented as mean $\pm \mathrm{SD}$, analyzed using one-way or two-way repeated measures ANOVA where appropriate, followed by the Student-Newman-Keuls post hoc test (SigmaStat, SPSS, Chicago, IL). $P$ values of $<0.05$ were considered statistically significant. 


\section{RESULTS}

Spectral analysis of the LDF data revealed CuBF oscillations associated with the heart rate and the respiratory rate in the higher frequency ranges. However, in the low frequency range (410 cycles/min; cpm), the flow-motion dominated the frequency spectrum, and the characteristic peak frequency could be determined in the various skin regions of most subjects in virtually all years (Table 1). The distal limb regions were notable exceptions: the occurrence of flow-motion in the crural region and in the forearm was quite sporadic, and therefore these regions were omitted from any further analysis. The characteristic flow-motion frequencies determined were essentially identical between the two sides (Fig. 2A), there was no significant side-difference in any studied region. The characteristic flow-motion frequencies showed remarkable stability (Fig. 2B), the values were usually unchanged in the three study years in all assessed regions, only in the infraclavicular region was there a small increase in the third year compared to the first. Interestingly, the characteristic flow-motion frequency displayed pronounced regional differences with a clear spatial pattern: CuBF flow-motion frequency was the highest in the two head regions, followed by the trunk regions, and finally the posterior femoral region (Fig. 2C).

Transient occlusion of the brachial artery resulted in occlusion time dependent increases in $\mathrm{CuBF}$, also known as PORH (Fig. 3A). During PORH, the peak CuBF values were significantly higher after 2-3 min compared to 1 min occlusion (Fig. 3B). Furthermore, the PORH indices showed clear occlusion time dependence, their values increased significantly with each increasing occlusion time (Fig. 3B). However, there was also a significant difference in the PORH indices obtained in the two study years at 2 and 3 min occlusion (Fig. 3B).
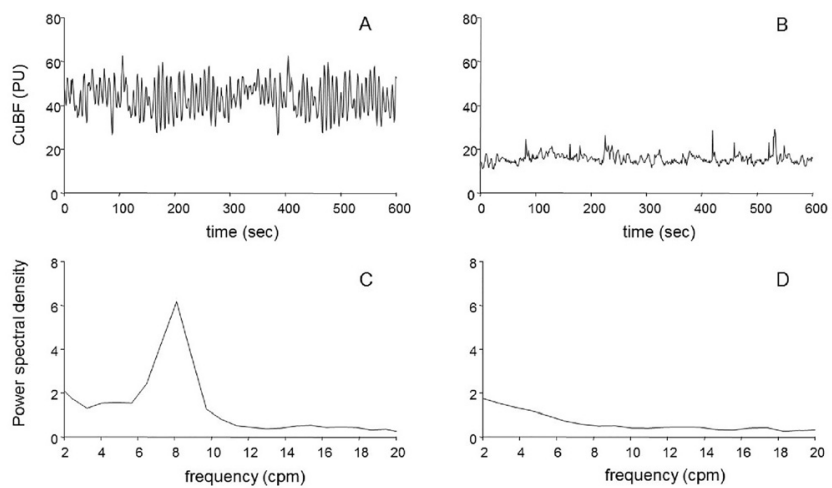

Fig. 1. Power spectral analysis of laser-Doppler signal to determine the characteristic frequency of flowmotion in the cutaneous microcirculation. Panels A, B: original, representative recordings of forehead (A) and crural (B) cutaneous blood flow (CuBF) obtained with laser-Doppler flowmetry (LDF) shown in perfusion units $(\mathrm{PU})$ in a female participant. Panels $\mathrm{C}, \mathrm{D}$ : power spectral density curves obtained from the respective LDF signals shown in Panels A, B. In the forehead region, a pronounced peak at $8.2 \mathrm{cycles} / \mathrm{min}$ $(\mathrm{cpm})$ indicates the characteristic flow-motion frequency (Panel C), whereas in the crural region flowmotion could not be detected (Panel D) 
Table 1. The occurrence of cutaneous vasomotion in the proband over the course of three consecutive years (1-3 y)

\begin{tabular}{|c|c|c|c|c|c|c|c|c|c|c|c|c|c|c|c|c|c|c|c|c|c|c|c|c|}
\hline & \multicolumn{3}{|c|}{ Forehead } & \multicolumn{3}{|c|}{ Temporal reg. } & \multicolumn{3}{|c|}{ Scapular reg. } & \multicolumn{3}{|c|}{$\begin{array}{l}\text { Infra- } \\
\text { clavicular } \\
\text { reg. }\end{array}$} & \multicolumn{3}{|c|}{ Coxal reg. } & \multicolumn{3}{|c|}{$\begin{array}{l}\text { Posterior } \\
\text { femoral reg. }\end{array}$} & \multicolumn{3}{|c|}{ Crural reg. } & \multicolumn{3}{|c|}{ Forearm } \\
\hline & $1 y$ & $2 y$ & $3 y$ & $1 \mathrm{y}$ & $2 y$ & $3 y$ & $1 y$ & $2 y$ & $3 y$ & $1 y$ & $2 y$ & $3 y$ & $1 y$ & $2 y$ & $3 y$ & $1 y$ & $2 y$ & $3 y$ & $1 y$ & $2 y$ & $3 y$ & $1 y$ & $2 y$ & $3 y$ \\
\hline Subject 1 & + & + & + & + & + & + & + & + & + & + & + & + & + & + & + & + & + & + & - & - & - & - & + & - \\
\hline Subject 2 & + & + & + & + & + & + & + & + & + & + & + & + & + & + & + & + & + & + & + & - & - & - & - & - \\
\hline Subject 3 & + & + & + & + & + & + & + & + & + & - & + & - & + & + & - & + & + & + & + & - & - & + & - & - \\
\hline Subject 4 & + & + & + & + & + & + & + & + & + & + & + & + & + & + & + & + & + & + & - & - & + & - & - & + \\
\hline Subject 5 & + & + & + & + & + & + & + & + & + & + & + & - & + & + & + & + & + & + & - & - & - & - & - & - \\
\hline Subject 6 & + & + & + & + & + & + & + & + & - & + & + & + & + & + & + & - & + & - & - & - & - & - & - & - \\
\hline
\end{tabular}



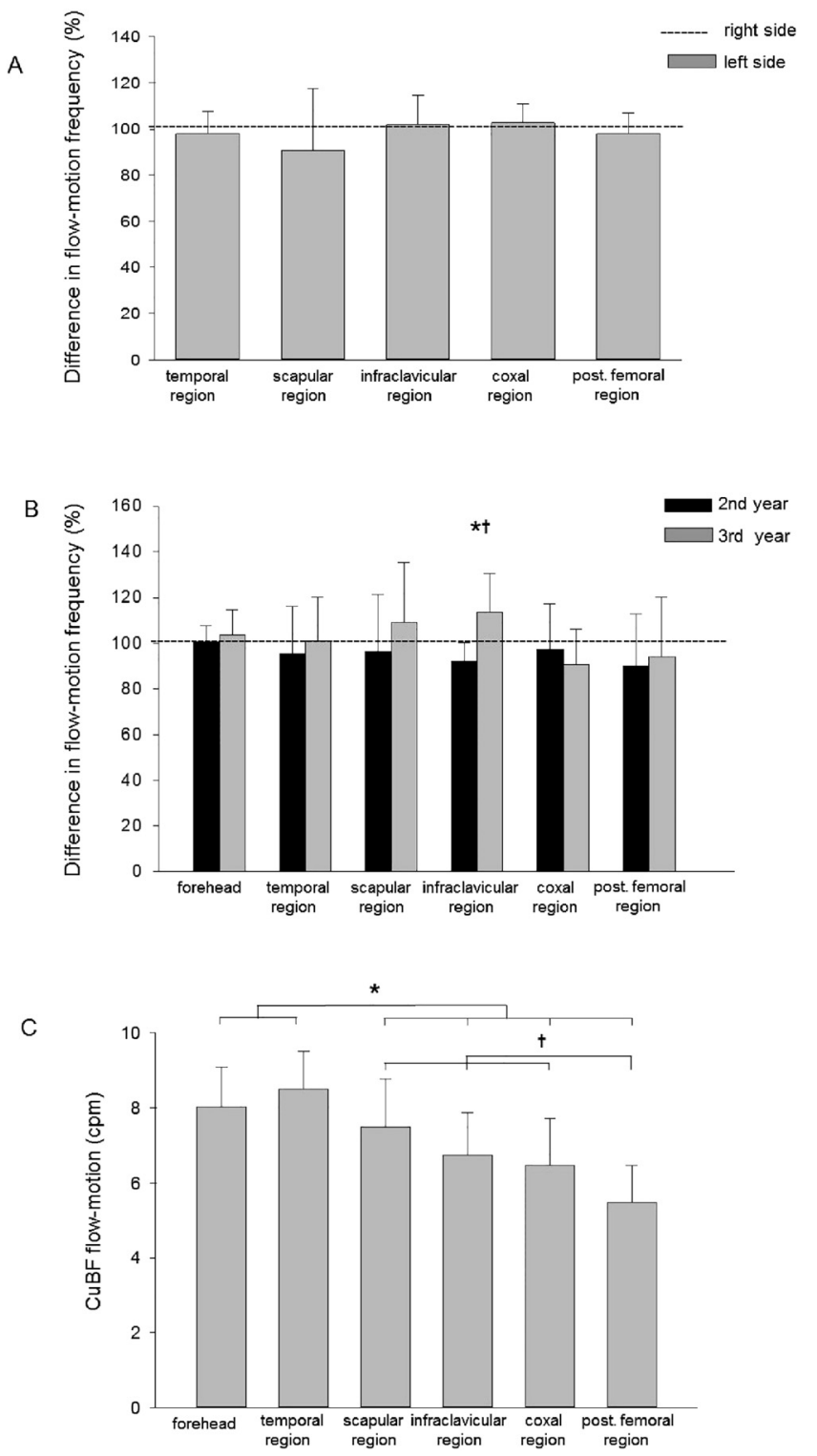

Fig. 2. Stability of region-dependent cutaneous blood flow-motion frequencies. Panel A: Flow-motion frequencies determined from either side of the body were remarkably similar, there was no significant difference of the characteristic frequency of flow-motion in any of the skin regions studied. Data are expressed as percent deviations from the respective flow-motion frequency determined in the contralateral region (dashed line). Panel B: Flow-motion frequencies over the course of the study period are conserved. Data are expressed as percent deviations from the respective flow-motion frequency determined in the first year (dashed line). There was no significant alteration of the characteristic flow-motion frequency in any region in the second year (black bars), and in the third year (gray bars) there was only one, the infr 


\section{DISCUSSION}

The major novel findings of the present study are the following: 1) low-frequency flow-motion is a region-dependent feature of the human cutaneous microcirculation with a clear spatial pattern; 2) flow-motion frequency shows better intra-individual temporal stability than the PORH index in healthy young volunteers.

The dominant flow-motion frequencies determined in the present study were all in the 3.5$10.1 \mathrm{cpm}(0.06-0.17 \mathrm{~Hz})$ frequency range. This frequency of flow-oscillations is accepted to originate in the vascular smooth muscle of the microcirculatory resistance vessels. The rhythmic oscillation of smooth muscle contractions results in rhythmic vascular diameter changes, also known as vasomotion. The contribution of vasomotion to the flow-motion has been previously established [15], however, the present study extends previous observations in several aspects. First, the present study examined vasomotion-related flow-motion in many skin regions, and collected novel spatial information on the occurrence of the vasomotion in healthy young human subjects. The study of regional differences between the skin microvascular responses has been limited, for instance restricted to the assessment of different regions on the foot (dorsum of foot versus ankle) [3]. Indeed, we have already previously demonstrated that vasomotion was prominent in the frontal region but virtually absent in the forearm skin [4]. On one hand, the present study confirmed these previous findings, but it also extended them by demonstrating that a clear cranio-caudal as well as a proximal-distal pattern emerges in the frequency of vasomotion. More specifically, not only the frontal but also the temporal skin areas possess the highest frequency of vasomotion. Moreover, the previously uncharted trunk regions also display pronounced vasomotion in most subjects, albeit with smaller frequencies. In the limbs, vasomotion becomes more erratic, but in the femoral regions an even further decrease in vasomotion frequency was still detectable. Importantly, we found the vasomotion pattern symmetrical, without side-differences in any of the studied regions. The underlying mechanism of the regionally different vasomotion frequencies is virtually unknown. Vasomotion is thought to be an intrinsic feature of vascular smooth muscle cells, where vasomotion develops as a consequence to the oscillation of $\mathrm{Ca}^{2+}$ ions induced by a cytoplasmic and/or a membrane oscillator [1]. It is intruiguing that perhaps distinct oscillator mechanisms with various frequencies may operate in the different skin regions as suggested by our present findings. The different regionspecific vasomotion phenotypes suggest that in the different skin regions, physiologically distinct vascular smooth muscle populations may exist.

Unexpectedly, the region-dependent vasomotion frequencies determined displayed a high degree of stability over the study years. Previous studies investigated the repeatability of CuBF microvascular responses recorded with LDF over much shorter time courses, usually over the course of a few days or weeks. For instance, microvascular reactivity assessed with PORH,

aclavicular region, where a minor, but statistically significant difference was observed $(P<0.05$, * versus first year, $\dagger$ versus second year). Panel C: the characteristic flow-motion frequencies show region-dependence. The statistical analysis identified three body regions: head, trunk and limb regions with significant differences in their flow-motion frequencies. The flow-motion has the highest frequency in the head regions (frontal and temporal regions), has significantly lower frequencies in the trunk regions (scapular, infraclavicular and coxal), and has the lowest frequency value in the femoral region $\left(P<0.05 .^{*}\right.$ versus head regions, $\uparrow$ versus trunk regions) 

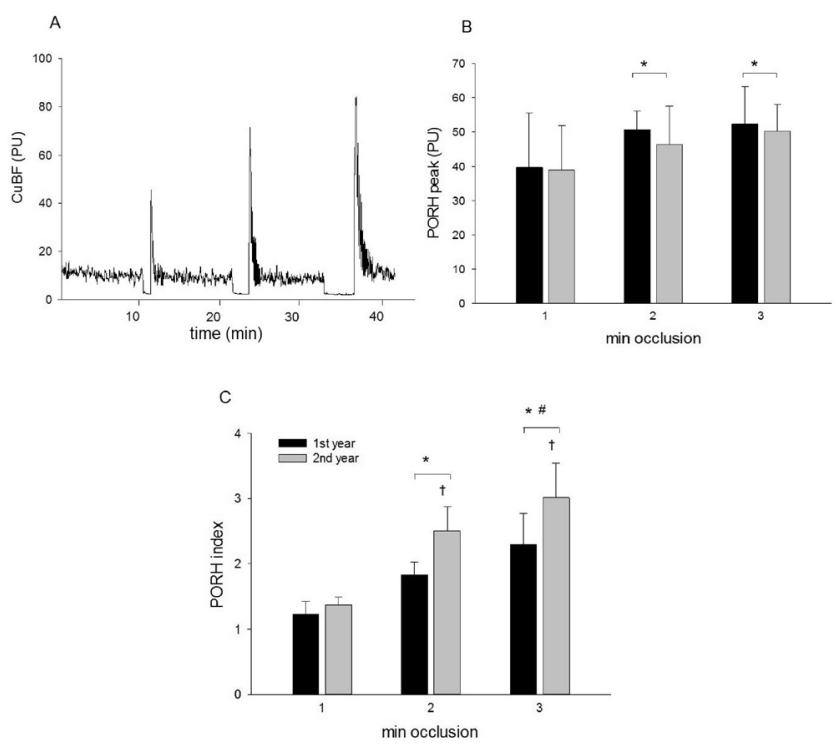

Fig. 3. Assessment of postocclusive reactive hyperemia (PORH). Panel A: original, representative recording of forearm cutaneous blood flow $(\mathrm{CuBF})$ obtained with laser-Doppler flowmetry (LDF) shown in perfusion units (PU) in a female participant before, during and after 1, 2, and 3 min of brachial artery occlusion induced by shypgmomanometer cuff inflation. Severely reduced perfusion during the occlusion periods and rapid onset of PORH can be observed. Panel B: peak CuBF during PORH were similar in the consecutive years, however, there was small but statistically significant difference between the peak PORH CuBF values after 1 and 2 or 3 min occlusion $\left(P<0.05^{*}\right.$ versus first year). Panel C: PORH indices determined by calculating the area under the CuBF curve showed significant increases with increasing occlusion times $(P<$ $0.05^{\star}$ versus 1 min occlusion, \# versus 2 min occlusion). Furthermore, there was a significant difference in the PORH indices obtained both after 2 or 3 min occlusions $(P<0.05 \dagger$ versus first year $)$

thermal hyperemia or acetyl-choline iontoforesis were found to be reliably repeatable in the short time-frame of the experiment $[5,13,16,24]$. The reproducibility of the above mentioned microvascular responses over similarly short study periods has also been demonstrated with other techniques such as laser-speckle contrast imaging [17] or flow-mediated skin fluorescence [10]. We are not aware of any previous studies assessing any aspect of skin microvascular function in the same subjects over the course of years. Despite the small size of the proband, we still found significant alterations in the PORH indices with the simultaneous demonstration of robust reproducibility of the region-specific characteristic vasomotion frequencies. In our study, we employed 1-2-3 min arterial occlusion that is known to evoke occlusion time dependent increases in PORH index [22]. We declined to use longer occlusion periods such as 5 minutes also employed by some previous studies [23], as these longer periods lead to discomfort and pain that might affect the results independently of microvascular function in agreement with Rossi et al. [14]. The PORH indices determined in the present study confirmed, on the one hand, the previously established occlusion time dependence. However, on the other hand, the PORH 
indices differed between the two study years. The cause of this difference is unknown; however, it is unlikely to be caused by reductions in microvascular reactivity, as the actual PORH indices were significantly higher in the second year. Unchanged microvascular function is also supported by the retained vasomotion in all of the assessed regions.

In summary, the non-invasive assessment of CuBF with LDF yielded novel observations on the physiology of human cutaneous microcirculation by establishing a robust, stable, regionspecific presence and frequency of vasomotion-related flow-motion. These findings imply that the yet unexplored vasomotion-related flow-motion could also be of use for characterizing microvascular function. Recent studies indicated that microvascular dysfunction assessed with PORH can be reversed by short-term weight loss in obese individuals [7], and it can even predict cognitive impairment in aging individuals [8]. Based on our present findings, future studies on aging are required to determine if the flow-motion frequencies are also affected in obesity- or

aging-related microvascular dysfunction, and if their stability in prospective studies may be superior to the PORH test.

Conflict of interest: The authors declare they have no conflict of interests.

\section{REFERENCES}

1. Aalkjaer C, Nilsson H. Vasomotion: cellular background for the oscillator and for the synchronization of smooth muscle cells. Br J Pharmacol 2005; 144: 605-16. https://doi.org/10.1038/sj.bjp.0706084.

2. Balasubramanian P, Hall D, Subramanian M. Sympathetic nervous system as a target for aging and obesityrelated cardiovascular diseases. Geroscience 2019; 41: 13-24. https://doi.org/10.1007/s11357-018-0048-5.

3. Balaz D, Komornikova A, Kruzliak P, Sabaka P, Gaspar L, Zulli A, et al. Regional differences of vasodilatation and vasomotion response to local heating in human cutaneous microcirculation. Vasa 2015; 44: 458-65. https://doi.org/10.1024/0301-1526/a000469.

4. Bari F, Toth-Szuki V, Domoki F, Kalman J. Flow motion pattern differences in the forehead and forearm skin: age-dependent alterations are not specific for Alzheimer's disease. Microvasc Res 2005; 70: 121-8. https://doi. org/10.1016/j.mvr.2005.09.001

5. Barwick A, Lanting S, Chuter V. Intra-tester and inter-tester reliability of post-occlusive reactive hyperaemia measurement at the hallux. Microvasc Res 2015; 99: 67-71. https://doi.org/10.1016/j.mvr.2015.03.001.

6. Benedicic M, Bernjak A, Stefanovska A, Bosnjak R. Continuous wavelet transform of laser-Doppler signals from facial microcirculation reveals vasomotion asymmetry. Microvasc Res 2007; 74: 45-50. https://oi.org/ 10.1016/j.mvr.2007.02.007.

7. Csipo T, Fulop GA, Lipecz A, Tarantini S, Kiss T, Balasubramanian P, et al. Short-term weight loss reverses obesity-induced microvascular endothelial dysfunction. Geroscience 2018; 40: 337-46. https://doi.org/10. 1007/s11357-018-0028-9.

8. Csipo T, Lipecz A, Fulop GA, Hand RA, Ngo BN, Dzialendzik M, et al. Age-related decline in peripheral vascular health predicts cognitive impairment. Geroscience 2019; 41: 125-36. https://doi.org/10.1007/s11357019-00063-5.

9. Farkas K, Kolossvary E, Jarai Z, Nemcsik J, Farsang C. Non-invasive assessment of microvascular endothelial function by laser Doppler flowmetry in patients with essential hypertension. Atherosclerosis 2004; 173: 97-102. https://doi.org/10.1016/j.atherosclerosis.2003.11.015. 
10. Hellmann M, Tarnawska M, Dudziak M, Dorniak K, Roustit M, Cracowski JL. Reproducibility of flow mediated skin fluorescence to assess microvascular function. Microvasc Res 2017; 113: 60-4. https://doi.org/ 10.1016/j.mvr.2017.05.004.

11. Hodges GJ, Del Pozzi AT. Noninvasive examination of endothelial, sympathetic, and myogenic contributions to regional differences in the human cutaneous microcirculation. Microvasc Res 2014; 93: 87-91. https://doi. org/10.1016/j.mvr.2014.04.002.

12. Kiss T, Balasubramanian P, Valcarcel-Ares MN, Tarantini S, Yabluchanskiy A, Csipo T, et al. Nicotinamide mononucleotide $(\mathrm{NMN})$ treatment attenuates oxidative stress and rescues angiogenic capacity in aged cerebromicrovascular endothelial cells: a potential mechanism for the prevention of vascular cognitive impairment. Geroscience 2019; 41: 619-30. https://doi.org/10.1007/s11357-019-00074-2.

13. Kubli S, Waeber B, Dalle-Ave A, Feihl F. Reproducibility of laser Doppler imaging of skin blood flow as a tool to assess endothelial function. J Cardiovasc Pharmacol 2000; 36: 640-8. https://doi.org/10.1097/00005344200011000-00014.

14. Rossi M, Bradbury A, Magagna A, Pesce M, Taddei S, Stefanovska A. Investigation of skin vasoreactivity and blood flow oscillations in hypertensive patients: effect of short-term antihypertensive treatment. J Hypertens 2011; 29: 1569-76. https://doi.org/10.1097/HJH.0b013e328348b653.

15. Rossi M, Carpi A, Di Maria C, Franzoni F, Galetta F, Santoro G. Post-ischaemic peak flow and myogenic flowmotion component are independent variables for skin post-ischaemic reactive hyperaemia in healthy subjects. Microvasc Res 2007; 74: 9-14. https://doi.org/10.1016/j.mvr.2007.02.006.

16. Roustit M, Blaise S, Millet C, Cracowski JL. Reproducibility and methodological issues of skin post-occlusive and thermal hyperemia assessed by single-point laser Doppler flowmetry. Microvasc Res 2010; 79: 102-8. https://doi.org/10.1016/j.mvr.2010.01.001.

17. Roustit M, Millet C, Blaise S, Dufournet B, Cracowski JL. Excellent reproducibility of laser speckle contrast imaging to assess skin microvascular reactivity. Microvasc Res 2010; 80: 505-11. https://doi.org/10.1016/j. mvr.2010.05.012.

18. Salerud EG, Tenland T, Nilsson GE, Oberg PA. Rhythmical variations in human skin blood flow. Int J Microcirc Clin Exp 1983; 2: 91-102.

19. Santesson P, Lins PE, Kalani M, Adamson U, Lelic I, von Wendt G, et al. Skin microvascular function in patients with type 1 diabetes: an observational study from the onset of diabetes. Diab Vasc Dis Res 2017; 14: 191-9. https://doi.org/10.1177/1479164117694463.

20. Stefanovska A, Bracic M, Kvernmo HD. Wavelet analysis of oscillations in the peripheral blood circulation measured by laser Doppler technique. IEEE Trans Biomed Eng 1999; 46: 1230-9. https://doi.org/10.1109/10. 790500.

21. Sure VN, Sakamuri S, Sperling JA, Evans WR, Merdzo I, Mostany R, et al. A novel high-throughput assay for respiration in isolated brain microvessels reveals impaired mitochondrial function in the aged mice. Geroscience 2018; 40: 365-75. https://doi.org/10.1007/s11357-018-0037-8.

22. Tee GB, Rasool AH, Halim AS, Rahman AR. Dependence of human forearm skin postocclusive reactive hyperemia on occlusion time. J Pharmacol Toxicol Methods 2004; 50: 73-8. https://doi.org/10.1016/j.vascn. 2004.02.002.

23. Yamamoto-Suganuma R, Aso Y. Relationship between post-occlusive forearm skin reactive hyperaemia and vascular disease in patients with Type 2 diabetes-a novel index for detecting micro- and macrovascular dysfunction using laser Doppler flowmetry. Diabet Med 2009; 26: 83-8. https://doi.org/10.1111/j.1464-5491. 2008.02609.x. 
24. Yvonne-Tee GB, Rasool AH, Halim AS, Rahman AR. Reproducibility of different laser Doppler fluximetry parameters of postocclusive reactive hyperemia in human forearm skin. J Pharmacol Toxicol Methods 2005; 52: 286-92. https://doi.org/10.1016/j.vascn.2004.11.003.

Open Access statement. This is an open-access article distributed under the terms of the Creative Commons Attribution 4.0 International License (https://creativecommons.org/licenses/by/4.0/), which permits unrestricted use, distribution, and reproduction in any medium, provided the original author and source are credited, a link to the CC License is provided, and changes - if any - are indicated. (SID_1) 